\title{
Atypical carcinoid: A rare finding of a man with mediastinal mass: A case report
}

\author{
WEI-XIA XUAN, JIN-JIN LI, YU-JIE SHI and XIAO-JU ZHANG \\ Department of Respiratory and Critical Care Medicine, Henan Provincial People's Hospital (People's Hospital of \\ Zhengzhou University), Zhengzhou, Henan 450003, P.R. China
}

Received April 11, 2019; Accepted January 29, 2020

DOI: $10.3892 / \mathrm{mco} .2020 .2001$

\begin{abstract}
The present case report describes a case of mediastinal atypical carcinoid and a favorable outcome linked with the treatment. Mediastinal atypical carcinoid is a rare and aggressive type of neuroendocrine tumor. A 56-year-old man was admitted at the Respiratory Department due to intermittent tightness of the chest for 1 month. An initial diagnosis of a mass in the left anterior mediastinum was conducted using CT scan and immunohistochemistry. Laboratory data revealed the following values: Neuron Specific Enolase of $62.13 \mathrm{ng} / \mathrm{ml}$ (reference range, 0-40 ng/ml); CYFRA21 of $3.01 \mathrm{ng} / \mathrm{ml}$ (reference range, 0-3.3 ng/ml); CEA of 4.22 (0-6.5) ng/ml; SCC of $0.5(0-1.5) \mathrm{ng} / \mathrm{ml}$; CA125 of 67.24 (0-35) U/ml; AFP of 23 (0-25) U/ml; CRP of 96.7 (0-10) mg/l; PCT $<0.05(0-0.05) \mathrm{ng} / \mathrm{ml}$; and ESR of $48(0-20) \mathrm{mm} / \mathrm{h}$. Tissue pathology revealed tumor cells with small cell pattern, and cell proliferation activity was $10 \%$. Combined chemotherapy with bevacizumab (0.4 g, qd, once every 21 days) and capecitabine $(0.15 \mathrm{~g}, \mathrm{Bid}, \mathrm{Po})$ and timozolamine $(0.34 \mathrm{mg}, \mathrm{qd}$, po) was administered for 6 cycles. After the patient was given chemotherapy, the symptoms and CT exhibited improvement. On March 11, 2018, the lesion progressed into the lymph and pleura. The patient was commenced on radiotherapy and new chemotherapeutic regimen etoposide $(0.5 \mathrm{~g})$-carboplatin $(0.4 \mathrm{~g})$-bevacate $(0.4 \mathrm{~g})$. Another CT scan was performed after a month which revealed a substantial decrease in tumor size. Hence, a CT scan was performed for this patient who further revealed a decrease in tumor size. Currently patients are treated with bevacizumab maintenance therapy. Further studies of conservative treatment of chemotherapy and radiotherapy may provide a treatment to improve atypical carcinoid.
\end{abstract}

Correspondence to: Professor Xiao-Ju Zhang, Department of Respiratory and Critical Care Medicine, Henan Provincial People's Hospital (People's Hospital of Zhengzhou University), 7 Weiwu Road, Zhengzhou, Henan 450003, P.R. China

E-mail: 15837101166@163.com

Key words: atypical carcinoid, mediastinal mass, dyspnea

\section{Introduction}

Mediastinal atypical carcinoid is a type of neuroendocrine tumor which is rare and aggressive mediastinal tumor (1). Neuroendocrine carcinoma is often divided into typical carcinoid, atypical carcinoid, small cell carcinoma and large cell neuroendocrine carcinoma based on morphology. Small cell carcinoma and large cell neuroendocrine carcinoma are high-grade tumors. Typical and atypical carcinoid tumors are low-medium grade tumors (2). Clinically, patients may be asymptomatic or show local symptoms because of the compression or invasion of mediastinal structures, or systemic symptoms secondary to the tumor ability to produce hormones or cytokines (3). Neuroendocrine tumors (NETs) are epithelial neoplasms with major neuroendocrine differentiation that begin in most organs of the body. Primary NET of the mediastinum are very occasional (2), they have been the source of investigation and debatable in the literature because of their origin.

Thymic carcinoids characterize by unusual neoplasms. Their yearly over all age-adjusted occurrence has been described to be estimated 1 of 10,000,000 (4), and roughly 200 cases have been stated in the literature (5). Thymic carcinoids occur mainly in men with a ratio of $3: 1$ to that of women. It has a middling age of 43 years (range 39-60 years)(6). Neuroendocrine carcinomas of the thymus and are reasonably differentiated with a high rate of metastasis. These tumors reveal a 5-year survival rate of $60 \%$ and their reappearance is common. $(7,8)$ Furthermore, the brutality of the illness is frequently abandoned since the medical appearance is somewhat benign. Hence, thymic carcinoids are usually revealed at a far advanced stage, which may also account for the poor prognosis (9)

Thymic carcinoids often act destructively. Thorough surgical removal of the thymus is the first treatment of high-quality for thymic carcinoids as chemotherapy and radiotherapy are not active for lengthening survival $(9,10)$. When the tumor is destructive to the patient, surgery is still the best way to eradicate the tumor for causing death. Different options have been raised on the functions of chemotherapy and radiotherapy in the postoperative management of thymic carcinoids. However, adjuvant treatment may also assist in the disease control, it is not active to eliminate tumors and to inhibit the growth of tumor or metastases (11). On the other 
A

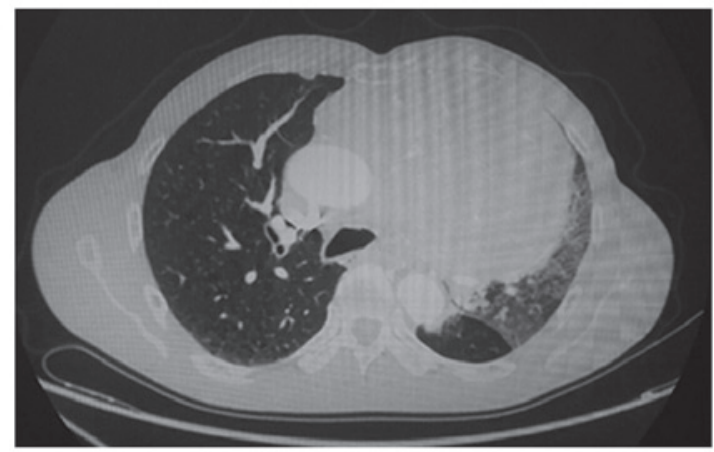

B

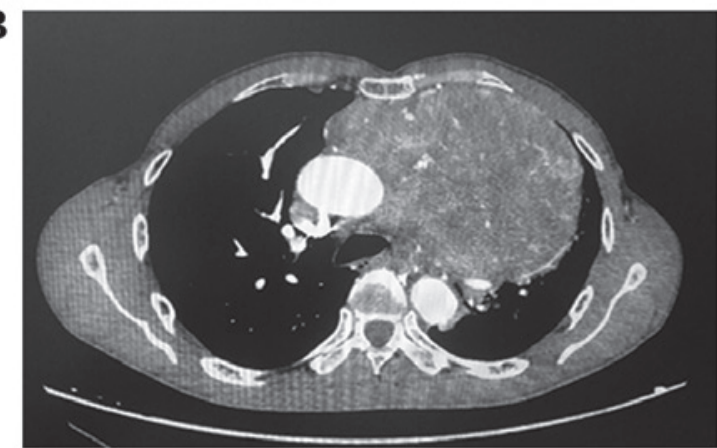

Figure 1. CT scan showing a mass of soft tissue density of $16.5 \times 13.0 \mathrm{~cm}$ in the left anterior mediastinum with a clear boundary and uneven density. The enhancement scan demonstrated uneven and distinct enhancement. There was no obvious fracture destruction in the adjacent bone. The adjacent trachea and bronchial cavity were compressed and narrowed, and the heart moved to the right. (A) Lung window. (B) Mediastinal window.
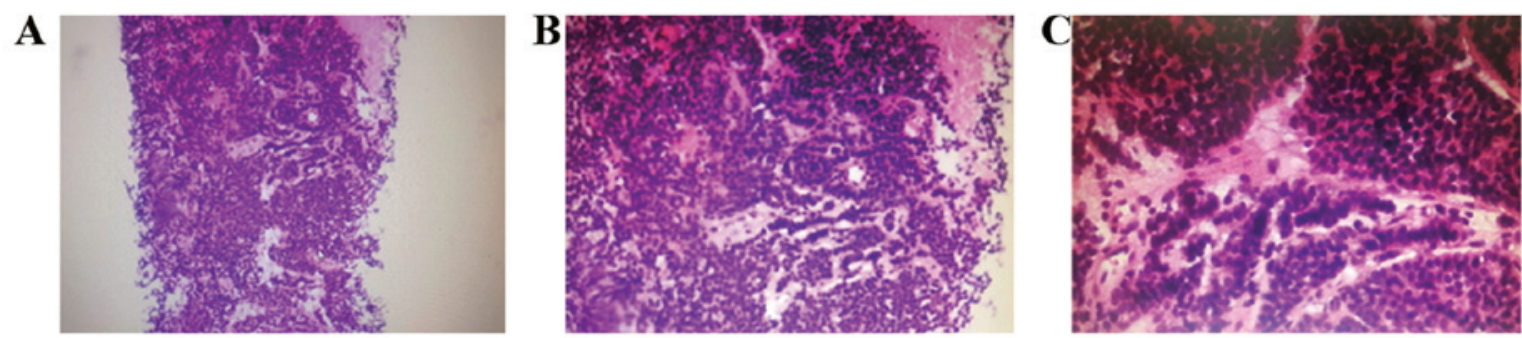

Figure 2. Hematoxylin and eosin staining. (A) Original magnification, x10. (B) Original magnification, x20. (C) Original magnification, x40.

hand, adjuvant radiotherapy has been described in avoidance of local-regional reappearance (12). Utilization of the following therapy only or in combination chemotherapies with 5-fluorouracil, streptozocin, carmustine, VP-16, and cisplatin have been administered earlier without any significant influence on the reappearance rate or overall survival (13). Herein we reported a very special case of primary cutaneous NET (atypical carcinoid) expressing CA125 and CRP with immunohistochemical markers (CD 56+, CD 117+, Syn+, $\mathrm{CgA}+$.and $\mathrm{CK}+)$.

\section{Case report}

A 56-year-old male was presented with intermittent chest tightness for 1 month. His chest tightness was aggravated with movement. He began to cough and developed hemoptysis after 11 days. The hemoptysis was a bright red and small amount. He did not have a history of chemicals, fumes, or dust exposure and no history of tobacco or alcohol abuse. Chest enhanced CT demonstrated a $16.5 \times 13.0 \mathrm{~cm}$ soft tissue mass in the left anterior mediastinum (Fig. 1). Laboratory data revealed the following values: Neuron Specific Enolase of $62.13 \mathrm{ng} / \mathrm{ml}$ (reference range, $0-40 \mathrm{ng} / \mathrm{ml}$ ), CYFRA21 of $3.01 \mathrm{ng} / \mathrm{ml}$ (reference range, 0-3.3 ng/ml), CEA of $4.22(0-6.5) \mathrm{ng} / \mathrm{ml}, \mathrm{SCC} 0.5(0-1.5) \mathrm{ng} / \mathrm{ml}$, CA125 67.24 (0-35) U/ml; AFP 23 (0-25) U/ml, CRP 96.7 mg/l (0-10); PCT <0.05 ng/ml (0-0.05); ESR $48 \mathrm{~mm} / \mathrm{h}(0-20)$. Tissue pathology revealed tumor cells with small cell pattern, cell proliferation activity was $10 \%$ (Fig. 2). Neuroendocrine carcinoma is characterized by invasive growth, lymphatic and blood metastasis. Therefore, it has been incorporated into low-grade malignancies by WHO. The cytoplasm of atypical carcinoid contains neuroendocrine granules, which has a secretory function and can lead to carcinoid syndromes such as paroxysmal skin flushing, diarrhea, asthma, tachycardia, and ectopic adrenocorticotropic syndrome. But these symptoms are not presented rare, and there is no appeal, in this case, however, thyroid, parathyroid and sex hormones assay were done and it was normal in this patient. The results of immunohistochemistry in this patient's tissue biopsy show: CD117(+), CD1a(-), CD5(-), CD56(+), CD99(-), $\mathrm{CgA}(+), \mathrm{CK}(\mathrm{AE} 1 / \mathrm{AE} 3)(+), \mathrm{CK} 19(+), \mathrm{Ki} 67(10 \%+), \mathrm{LCA}(-)$, SYN(+), TdT(-) TTF-1(-). Synaptophysin (Syn), chromaffin (CgA) and CD56 are the immunohistochemical markers for the diagnosis of neuroendocrine tumors (Figs. 3 and 4), recent research shows that $\mathrm{Ki}-67$ is a potentially meaningful marker for sub-categorization of lung neuroendocrine tumors, the cut-off value of $\mathrm{Ki} 67$ for typical/atypical carcinoids was $7.5 \%$ with sensitivity and specificity of 91.4 and $100 \%$ (area under curve is 0.9685) (14). CK19 assisted diagnosis of thymic epithelial origin, TTF-1 assisted diagnosis of lung and thyroid tissue origin. Therefore, the mediastinal mass is considered as a neuroendocrine tumor originates from the thymus. Considering that a tumor had been transferred to the pleural and multiple lymph nodes. We decided to have surgical excision after combined chemotherapy with bevacizumab + capecitabine + timozolamine. After the patient was given chemotherapy, the symptom and CT showed improvement but the patient and his family rejected surgery. On March 11, 2018 the lesion progressed into the lymph and pleura. Patient was commenced on radiotherapy and new chemotherapeutic regimen (etoposide-carboplatin-bevacate). Another CT scan was performed after a month which revealed a substantial decrease in tumor size (Fig. 5). Subsequently CT scan was performed for this patient which further revealed a decrease 

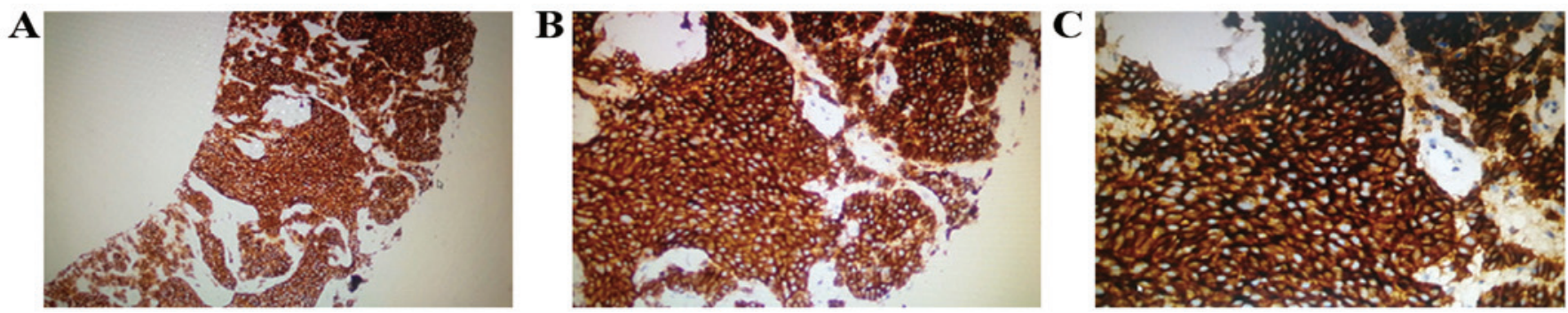

Figure 3. CD56 staining. (A) Original magnification, x10. (B) Original magnification, x20. (C) Original magnification, x40.
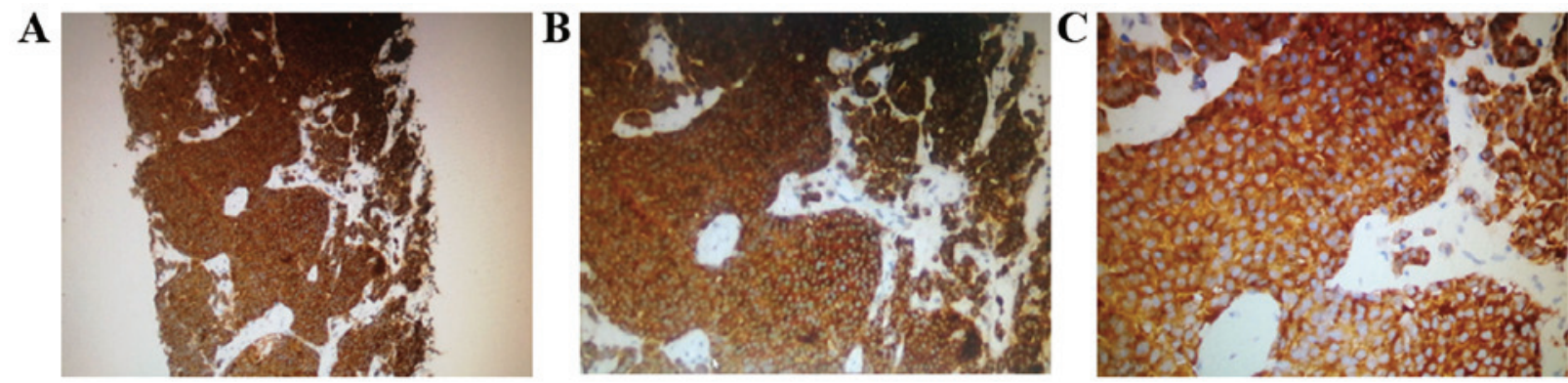

Figure 4. Synaptophysin staining. (A) Original magnification, x10. (B) Original magnification, x20. (C) Original magnification, x40.

A

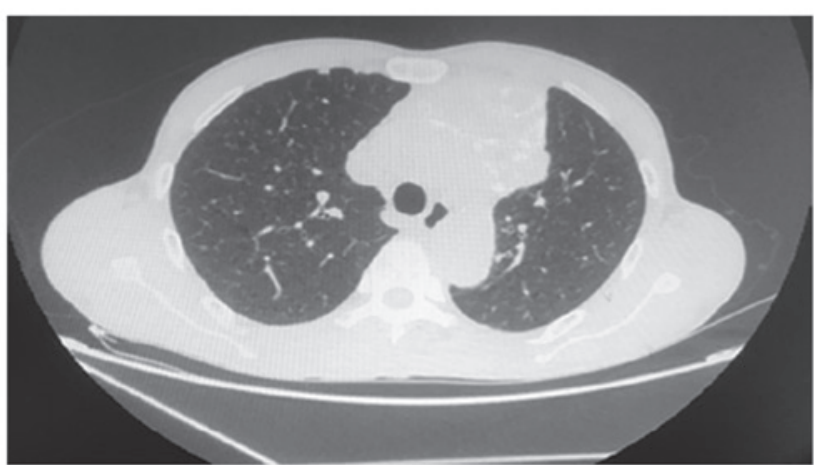

B

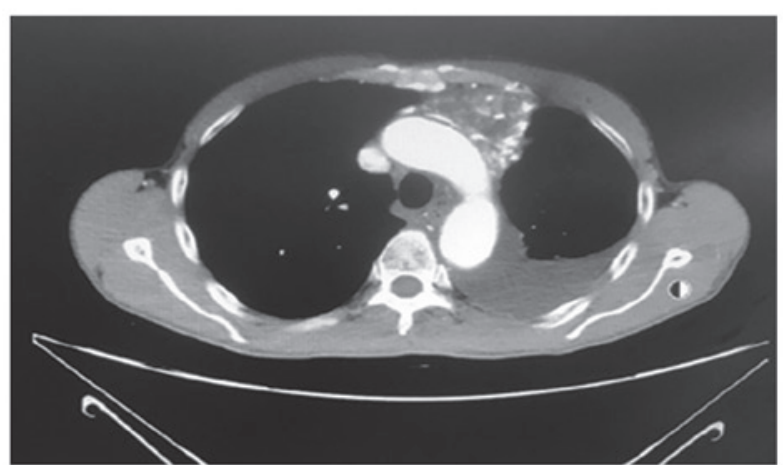

Figure 5. Alterations in CT scan after mediastinal radiotherapy and chemotherapy. Inflammation of the lungs, a few old lesions; limited thickening and adhesion of the bilateral pleura; a small amount of effusion in the pericardium; pleural effusion on the left side and local lung; insufficient tissue expansion; T8 vertebral body nodules, L1 vertebral body flattened, upper edge rough. (A) Lung window. (B) Mediastinal window.

in tumor size. Currently, patients have been treated with bevacizumab maintenance therapy.

\section{Discussion}

Atypical carcinoid may occur in many organs and tissues. They are more usually found in the gastro-entero-hepatic or respiratory system $(15,16)$. NETs of the mediastinum are very uncommon and usually, thymic NETs are the most common they are totally found in the anterosuperior mediastinum and account for 2 to $4 \%$ of all mediastinal tumors (17). Mediastinal NETs have been the foundation of considerable care and disagreement among experts and investigators because of their origin, that is still debatable. Their terminology and grouping have evolved over the years. Likened with bronchopulmonary carcinoid, primary NETs of the mediastinum are described by a poor prognosis due to their high tendency for local reappearance and previous distant metastases (13). The function of chemotherapy is disputed; in this case chemotherapy was used for the patient and it caused a reduction of tumor size and a decrease of proliferation rate. In the previous cases, of mediastinal NETs successfully treated by a combination of chemotherapy and Y-DOTATOC (18) or by radiotherapy alone (19) have been described. Hence, we utilized both chemotherapy and radiotherapy. Various investigators debated that a co-expression of CD117 (c-kit) and CD5 could be indicative of a neoplasm of thymic origin (20). In this case report, only CD117 was positive, thus not signifying a thymic origin. CD117 is frequently positive in small cell lung carcinoma and large cell NET and it is frequently negative in typical and atypical carcinoid. The positivity detected for c-kit could have vital therapeutic implication, such as the administration of etoposide $(0.5 \mathrm{~g})$-carboplatin $(0.4 \mathrm{~g})$-bevacate $(0.4 \mathrm{~g})$. The prognosis of atypical carcinoid is worse than that of typical carcinoid. Hence, it requires a multidisciplinary team approach for optimal treatment. This patient presented with 
pleural effusion. CT was progressive and the primary tumor shrank. But the patient feels that the general situation is okay and cannot be operated. It was first discovered in the late stage, with lymph node metastasis and pleural metastasis. We believe that further study of conservative treatment of chemotherapy and radiotherapy is a treatment to improve atypical carcinoid.

\section{Acknowledgements}

The authors would like to thank Dr Ayobami Olajuyin and Dr Adefunke Olajuyin (Department of Respiratory and Critical Care Medicine, The Provincial Hospital of Zhengzhou University, Zhengzhou, Henan, China) for helpful comments and revision of the manuscript.

\section{Funding}

The study was supported by the grant from Henan Provincial Department of Science and Technology (no. 182102310168 to WXX). The authors appreciate Dr Ayobami Olajuyin and Dr Adefunke Olajuyin (Department of Respiratory and Critical Care Medicine, the People's Hospital of Zhengzhou University) for helpful comments and significant revision of the manuscript.

\section{Availability of data and materials}

All data generated or analyzed during this study are included in this published article or are available from the corresponding author on reasonable request.

\section{Authors' contributions}

$\mathrm{XJZ}$ conceived the project and designed the experiments. WXX, JJL and YJS performed the experiments. WXX analyzed the data. WXX and XJZ wrote the paper. All authors read and approved the final manuscript.

\section{Ethics approval and consent to participate}

The study was approved by the Ethics Committee of Henan Provincial People's Hospital (Zhengzhou, China).Written informed consent was obtained from all patients.

\section{Patient consent for publication}

Not applicable.

\section{Competing interests}

The authors declare that they have no competing interests.

\section{References}

1. Melosky B: Advanced typical and atypical carcinoid tumours of the lung: Management recommendations. Curr Oncol 25: S86-S93, 2018.

2. Rindi G, Klersy C, Inzani F, Fellegara G, Ampollini L, Ardizzoni A, Campanini N, Carbognani P, De Pas TM, Galetta D, et al: Grading the neuroendocrine tumors of the lung: An evidence-based proposal. Endocr Relat Cancer 21: 1-16, 2013.
3. Ventura L, Gnetti L, Silini EM, Rindi G, Carbognani P, Rusca $\mathrm{M}$ and Ampollini L: Primary atypical carcinoid tumor of the mediastinum: A very rare finding. J Thorac Dis 9: 367-372, 2017.

4. Strosberg JR, Berry MF and Tanzelaar HD: Thymic neuroendocrine (carcinoid) tumors. UpToDate. https://www.uptodate. com/contents/thymic-neuroendocrine-carcinoid-tumors. Jul 23, 2019.

5. Gielda BT, Peng R, Coleman JL, Thomas CR and Cameron RB: Treatment of early stage thymic tumors: Surgery and radiationtherapy. Current treatment options in oncology 9: 259-268, 2008

6. Wick MR, Scott RE, Li CY and Carney JA: Carcinoid tumor of the thymus: A clinicopathologic report of seven cases with a review of the literature. Mayo Clin Proc 55: 246-254, 1980.

7. Tiffet O, Nicholson AG, Ladas G, Sheppard MN and Goldstraw P: A clinicopathologic study of 12 neuroendocrine tumors arising in the thymus. Chest 124: 141-146, 2003.

8. Vinik AI, Silva MP, Woltering EA, Go VLW, Warner R and Caplin M: Biochemical testing for neuroendocrine tumors. Pancreas 38: 876-889, 2009.

9. Lin FCF, Lin CM, Hsieh CC, Li WY and Wang LS: Atypical thymic carcinoid and malignant somatostatinoma in type I multiple endocrine neoplasia syndrome: Case report. Am J Clin Oncol 26: 270-272, 2003

10. John LC, Hornick P, Lang S, Wallis J, Edmondson SJ: Giant thymic carcinoid. Postgrad Med J 67: 462-465, 1991.

11. Fukai I, Masaoka A, Fujii Y, Yamakawa Y, Yokoyama T, Murase T, and Eimoto: Thymic neuroendocrine tumor (thymic carcinoid): A clinicopathologic study in 15 patients. Ann Thorac Surg 67: 208-211, 1999

12. Dutta R, Kumar A, Julka PK, Mathur SR, Kaushal S, Kumar R, Jindal T and Suri V: Thymic neuroendocrine tumour (carcinoid): Clinicopathological features 188 of four patients with different presentation. Interact Cardiovasc Thorac Surg 11: 732-736, 2010.

13. Gal AA, Kornstein MJ, Cohen C, Duarte IG, Miller JI and Mansour KA: Neuroendocrine tumors of the thymus: A clinicopathological and prognostic study. Ann Thorac Surg 72: 1179-1182, 2001.

14. Garg R, Bal A, DAS A, Singh N, Singh H: Proliferation marker (Ki67) in sub-categorization of neuroendocrine tumours of the lung. Turk Patoloji Derg 35: 15-21, 2019.

15. Caplin ME, Baudin E, Ferolla P, Filosso P, Garcia-Yuste M, Lim E, Oberg K, Pelosi G, Perren A and Rossi RE: Pulmonary neuroendocrine (carcinoid) tumors: European neuroendocrine tumor society expert consensus and recommendations for best practice for typical and atypical pulmonary carcinoids. Ann Oncol 26: 1604-1620, 2015.

16. Filosso PL, Guerrera F, Evangelista A, Welter S, Thomas P, Casado PM, Rendina EA, Venuta F, Ampollini L and Brunelli A: Prognostic model of survival for typical bronchial carcinoid tumours: Analysis of 1,109 patients on behalf of the European Association of Thoracic Surgeons, (ESTS) Neuroendocrine Tumours Working Group. Eur J Cardiothorac Surg 48: 441-447, 2015.

17. Franco R, Marino FZ, and Giordano A: The Mediastinal Mass-A multidisciplinary approach: 2018. Springer Link

18. Fazio N, Grana C, Pelosi G, Torrisi R, Di Meglio G, Tradati N, Lorizzo K and De Braud F: Successful chemotherapy and $90 Y-D O T A T O C$ in a patient with mediastinal highly aggressive neuroendocrine carcinoma. Acta Oncol 45: 627-629, 2006.

19. Furuta M, Hayakawa K, Kato S, Mitsuhashi N, Nakajima T and Niibe H: Malignant neuroendocrine tumor presenting a huge mediastinal mass controlled with radiation therapy. Lung cancer 22: 55-58, 1998.

20. Kriegsmann M, Muley T, Harms A, Tavernar, Goldmann T, Dienemann H, Herpel E and Warth A: Differential diagnostic value of $\mathrm{CD}_{5}$ and $\mathrm{CD}_{117}$ expression in thoracic tumors: $\mathrm{A}$ large scale study of 1,465 non-small cell lung cancer cases. Diagn Pathol 10: 210, 2015. 